\title{
Transient regime in non-linear transport through many-level quantum dots
}

\author{
Valeriu Moldoveanu, ${ }^{1}$ Vidar Gudmundsson, ${ }^{2}$ and Andrei Manolescu ${ }^{1}$ \\ ${ }^{1}$ National Institute of Materials Physics, P.O. Box MG-7, Bucharest-Magurele, Romania \\ ${ }^{2}$ Science Institute, University of Iceland, Dunhaga 3, IS-107 Reykjavik, Iceland
}

\begin{abstract}
We investigate the nonstationary electronic transport in noninteracting nanostructures driven by a finite bias and time-dependent signals applied at their contacts to the leads. The systems are modelled by a tight-binding Hamiltonian and the transient currents are computed from the non-equilibrium Green-Keldysh formalism. The numerical implementation is not restricted to weak coupling to the leads and does not imply the wide-band limit assumption for the spectral width of the leads. As an application of the method we study in detail the transient behavior and the charge dynamics in single and double quantum dots connected to leads by a step-like potential, but the method allows as well the consideration of non-periodic potentials or short pulses. We show that when the higher energy levels of the isolated system are located within the bias window of the leads the transient current approaches the steady state in a non-oscillatory smooth fashion. At moderate coupling to the leads and fixed bias the transient acquires a step-like structure, the length of the steps increasing with the system size. The number of levels inside a finite bias window can be tuned by a constant gate potential. We find also that the transient behavior depends on the specific way of coupling the leads to the mesoscopic system.
\end{abstract}

PACS numbers: 73.23.Hk, 85.35.Ds, 85.35.Be, 73.21.La

\section{INTRODUCTION}

The dynamics of conduction electrons in open nanostructures modulated by time-dependent signals is an outstanding problem in quantum transport theory. Extensive experimental and theoretical work has been done especially on quantum pumping, $1,2,3,4,5,6$ (a detailed bibliography can be found in the recent review $\left.{ }^{\underline{7}}\right)$ and photonassisted tunneling (see Ref. 8 and references therein). In these phenomena one is interested in measuring or computing the current response of a mesoscopic system driven by time-dependent periodic signals applied either on the system or on the attached leads. In particular, an unbiased system subjected to two periodic potentials differing by a phase lag generates a nonvanishing pumped current, provided one averages over the relevant period $\underline{\underline{4}}$ If the signal frequency is small the pumping is adiabatic and can be described by a 'frozen' scattering matrix as is rigorously shown in Ref. 9. A photon-assisted tunneling implies instead high frequencies and the measured current displays satellite peaks due to the additional sidebands $\underline{\underline{10}}$

From the theoretical point of view, any calculation of the current starts from defining the initial equilibrium state of the system and the perturbation that drives it. One way is to start with the connected system in the absence of the bias, and then to apply the bias adiabatically performing linear response calculations for the steady state current. An alternative picture was proposed by Caroli11 that takes as the equilibrium state the state of the decoupled system with the bias already imposed on the leads. The perturbation here is instead the coupling to the leads that is usually adiabatically switched in the remote past and eventually reaches its full magnitude at $t=0$. If one assumes that a steady state is achieved, the Green functions depend only on time differences and the Keldsyh formalism gives the corresponding current in terms of Fourier transformed quantities. Both pictures were shown to be useful in capturing and explaining important effects.

As for an ac signal, it can be included in the GreenKeldysh approach as a time-dependent global shift of the spectrum of the leads. 12 Note that the occupation probability (i.e. the Fermi function) of the leads stays timeindependent. Therefore this procedure assumes somehow that an ac signal is applied as well adiabatically. In the quantum pumping calculations the pumping potential is applied to the system in a steady-state, be it subjected to a finite bias or not. Finally the current (transient, timeaveraged or steady-state) is computed from the KeldyshGreen function formalism. $\underline{13}^{13}$

Here we aim to get some insight into a related topic: the calculation of the transient current through a quantum dot (QD) whose coupling to the leads is time dependent while the bias applied on the leads is constant. There are considerably fewer theoretical results on this issue (see the references below) and we were motivated also by recent increasing interest in using suitable electric pulses to investigate relaxation processes in quantum dots by using pump and probe measurements or transient current spectroscopy 14 As in the well known case of a turnstile pump $\frac{15}{5}$ these techniques imply oscillating tunnel barriers so that the transport formalism should deal with the nonlocal time-dependent coupling between the leads and the system.

The problem we want to look at is defined as follows: i) The system is disconnected at any time $t<0$, and the leads are submitted to a constant bias which is included through the difference between the chemical potentials of the leads. ii) At $t=0$ the leads are suddenly plugged to the system. Physically this means that the tunneling barriers between the leads and the system are set to be very 
high at $t<0$ and drop suddenly at $t=0$ to an intermediate value that allows charge transfer across the system. The simplest case of a constant barrier height at any $t>0$ opens already the problem of the existence of nonequilibrium steady-states in the long time limit. In general and at a rigorous level, one can prove the existence of such states when $t \rightarrow \infty \underline{16}$ and moreover, a Landauer formula was shown to hold for the steady-state current $\underline{\underline{17}}$ The method we developed is able to check the passage from transient behavior to steady state regime for specific systems, like many-level one- and two-dimensional quantum dots. As we shall see, the onset of the steady state for a given system depends on the its structure (number of levels), on the measurement setup (the strength of the coupling to the leads and the location of the contacts), and also on external parameters like gate potentials.

Although, in the present work the numerical simulations are restricted to the step-like coupling to the leads, our model allows the consideration of more general timedependent potentials between the leads and the central region. In particular we can investigate the response of a system to nonlocal time-dependent perturbations that can be switched on and off individually.

Recently there have been several theoretical approaches to the transient regime. In Ref. 19 the timedependent density functional was used to compute the transient current in a one-dimensional system submitted to a finite bias applied on the leads. The coupling term does not depend on time and the system is in an equilibrium state in the absence of the bias. Starting from this state the Kohn-Sham equation is used to calculate the response of the system to the external bias. The same techniques allow the calculation of time-averaged current in one-dimensional quantum pumps $\stackrel{20}{\underline{ }}$ On the other hand, Maciejko et al $\stackrel{18}{\underline{1}}$ have computed within the Keldysh framework the response of a single-site dot for a step-like or periodic signal applied to the leads, beyond the wide band limit. In their approach the computation of the time-dependent Green functions of the perturbed system uses steady-state Green functions of the coupled and biased system that have to be provided from density functional theory. We believe that theoretical results regarding the transient regime induced by time-dependent couplings of many-level systems would complement these results.

The content of the paper is organized as follows: Section II presents the model and the theoretical tools we use to compute the transient current. We rely essentially on the non-equilibrium Green-Keldysh formalism. However, in contrast to most of the previous studies we allow a complex structure for the central region coupled to leads (i.e. there is more than one single localized level and the system can be as complicated as we want: a single dot, a double dot or an Aharanov-Bohm interferometer). Also we go beyond the wide-band limit approximation and we solve exactly the integral Dyson equation for the retarded Green function of the coupled central region by a suitable numerical procedure. In doing so we take into account all the scattering processes between the leads and the sample.

Although the electron-electron interaction could presumably play an important role in the transient behavior and the formalism we use allows the inclusion of Coulomb terms in the Hamiltonian, we do not take it into account in this work. As is well known, the problem of the Coulomb interaction in the Keldysh approach is mainly technical and implies suitable approximation schemes for the interaction self-energy. We postpone this issue for future work. Section III gives extensive discussion of the numerical simulations for single and double dots. Section IV concludes the paper.

\section{FORMALISM}

The systems we study in this work have a typical transport configuration: a central region $(S)$ coupled to two semiinfinite leads $(\alpha$ and $\beta$ ) via a tunneling term (see Fig. 1 for a schematical representation). We shall use a tight-binding (TB) description of the Hamiltonian which has the following form:

$$
H(t)=H_{S}+H_{L}+H_{T}(t)
$$

where $H_{S}$ describes the system, $H_{L}$ the semiinfinite leads and $H_{T}(t)$ is the time-dependent tunneling term:

$$
H_{T}(t)=\sum_{\gamma=\alpha, \beta} \sum_{i \in \gamma} \sum_{m \in S} V_{i m}(t)\left(c_{i}^{\dagger} d_{m}+h . c\right) .
$$

Here $c_{i}$ and $c_{i}^{\dagger}$ denote the annihilation/creation opera-

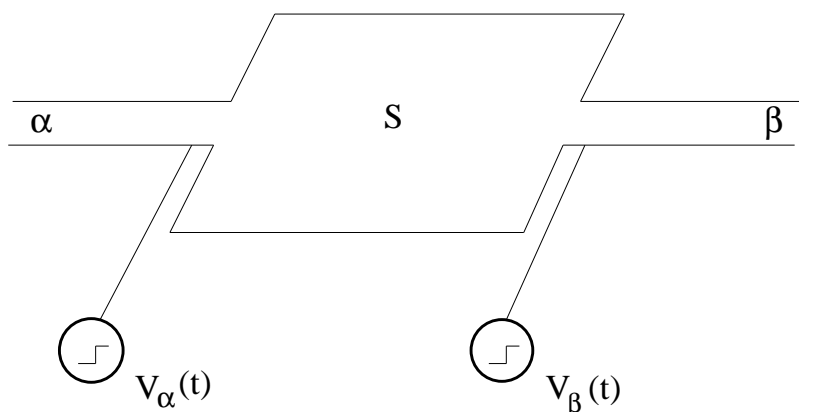

FIG. 1: Schematic picture of the system. The step-like potential is applied between the leads and the central region $S$ at $t=0$.

tors on the $i$-th site of the lead $\gamma$. Similarly $d_{m}$ and $d_{m}^{\dagger}$ is the pair of operators corresponding to the $m$-th site from the central region $S . V_{i m}(t)$ is the time-dependent hopping coefficient between the $i$-th site of the lead $\gamma$ and the $m$-th site of the central region. We take here a nearest-neighbor coupling so the double sums in the above expression contain only pairs of sites from the leads endpoint and the corresponding contact region from the central system:

$$
V_{i m}(t)= \begin{cases}V_{\gamma}(t) & \text { if } i, m \text { nearest neighbors } \\ & 0 \quad \text { otherwise }\end{cases}
$$


In this work we consider a steplike potential, i.e. $V_{\gamma}(t)=$ $V_{\gamma}$ if $t>0$ and zero otherwise. $H_{S}$ has a usual tightbinding form

$$
H_{S}=\sum_{m=1}^{N}\left(\epsilon_{m}+V_{g}\right) d_{m}^{\dagger} d_{m}+\sum_{\langle m, n\rangle} t_{m n} d_{m}^{\dagger} d_{n} .
$$

Here $t_{m n}$ are hopping terms, $\langle m, n\rangle$ denotes nearestneighbor summation over the system sites. $\epsilon_{m}$ is the onsite energy and the diagonal term $V_{g}$ simulates a plunger gate potential applied on the system. $N$ is the number of sites in the central region. The spectral width of the tight-binding lead is as usual $w:=\left[-2 t_{L}, 2 t_{L}\right]$, where $t_{L}$ is the hopping energy on leads (we take the same hopping constant on every lead). In the numerical calculation we choose $t_{L}$ such that it covers entirely the spectrum of the central region but we do not assume it to be infinite, as it is done in the wide-band limit approximation.

The central problem in electronic transport is to compute the statistical average of the time-dependent current operator in a given lead (say $\alpha) J_{\alpha}(t)=\operatorname{Tr}\left\{\rho(t) j_{\alpha}(t)\right\}$ using the statistical operator $\rho(t)$. Notice that the timedependence of the current operator appears only because of the time-dependent coupling. Denoting by $\left\{i_{\alpha}\right\}$ the endpoint sites of the lead $\alpha$ which are coupled to the sites $\left\{m_{\alpha}\right\}$ of the central region and by $M$ the number of sites in the transverse direction of the lead, the current operator $j_{\alpha}$ has the form (we take the electron charge as $-e$ and $e>0)$ :

$$
j_{\alpha}(t)=\frac{i e}{\hbar} \sum_{i_{\alpha}=1}^{M} \sum_{m_{\alpha} \in C_{\alpha}} V_{i_{\alpha} m_{\alpha}}(t)\left(c_{i_{\alpha}}^{\dagger} d_{m_{\alpha}}-d_{m_{\alpha}}^{\dagger} c_{i_{\alpha}}\right) .
$$

Since the statistical operator $\rho(t)$ of the coupled system is not easy to compute it is useful to move the timedependence entirely to the current operator by writing $\rho(t)$ in terms of the equilibrium statistical operator $\rho_{0}$ of the disconnected system. In general, the coupling to the leads is established at a given instant $t_{0}$ so that $\rho(t)=\rho_{0}$ for $t<t_{0}$. Then using the unitary evolution $\tilde{U}$ of the full Hamiltonian in the interaction picture w.r.t. the unperturbed one the solution of the quantum Liouville equation is given by

$$
\rho(t)=e^{-i t\left(H_{S}+H_{L}\right)} \tilde{U}\left(t, t_{0}\right) \rho\left(t_{0}\right) \tilde{U}\left(t, t_{0}\right)^{*} e^{i t\left(H_{S}+H_{L}\right)}
$$

Then it can be shown (see e.g. Ref. 22) that:

$$
J_{\alpha}(t)=\operatorname{Tr}\left\{\rho_{0} T_{C}\left(e^{-i \int_{C} d s \tilde{H}_{T}(s)} \tilde{j}_{\alpha}(t)\right)\right\},
$$

where $T_{C}$ is the ordering operator on the SchwingerKeldysh contour $C$ that runs from $t_{0}$ to $t$ and back to $t_{0}$. We remind the reader that in the case of adiabatic coupling the statistical operator becomes time-independent only in the remote past $t_{0} \rightarrow-\infty$. Both the coupling and current operators are written in the interaction picture. Using the definitions of the lesser Green functions in terms of the Heisenberg operators:

$$
\begin{aligned}
& G_{m_{\alpha} i_{\alpha}}^{<}\left(t, t^{\prime}\right)=i\left\langle c_{i_{\alpha}}^{\dagger}\left(t^{\prime}\right) d_{m_{\alpha}}(t)\right\rangle \\
& G_{i_{\alpha} m_{\alpha}}^{<}\left(t, t^{\prime}\right)=i\left\langle d_{m_{\alpha}}^{\dagger}\left(t^{\prime}\right) c_{i_{\alpha}}(t)\right\rangle,
\end{aligned}
$$

it follows that the current is given by a simpler relation:

$$
J_{\alpha}(t)=\frac{2 e}{\hbar} \sum_{i_{\alpha}=1}^{M} \sum_{m \in C_{\alpha}} \operatorname{Re}\left(V_{i_{\alpha} m_{\alpha}}(t) G_{m_{\alpha} i_{\alpha}}^{<}(t, t)\right) .
$$

At this point the standard Keldysh formalism requires the application of the so called Langreth rules 13 in order to express the lesser Green function $G_{m_{\alpha} i_{\alpha}}^{<}$in terms of the Green functions of the central region in the presence of the leads $G_{m_{\alpha} n_{\alpha}}^{<, R}$ and the Green functions of the isolated semiinfinite lead $g_{i_{\alpha} j_{\alpha}}^{<, A}$. The latter can be analytically computed:

$$
\begin{aligned}
& g_{i_{\alpha}, j_{\alpha}}^{A}\left(t, t^{\prime}\right)=i \theta\left(t^{\prime}-t\right) \sum_{p=1}^{M} \chi_{p}\left(i_{\alpha}\right) \chi_{p}\left(j_{\alpha}\right) \int_{-2 t_{L}+E_{p}}^{2 t_{L}+E_{p}} d E \rho\left(E-E_{p}\right) e^{-i E\left(t-t^{\prime}\right)} \\
& g_{i_{\alpha}, j_{\alpha}}^{<}\left(t, t^{\prime}\right)=i \sum_{p=1}^{M} \chi_{p}\left(i_{\alpha}\right) \chi_{p}\left(j_{\alpha}\right) \int_{-2 t_{L}+E_{p}}^{2 t_{L}+E_{p}} d E \rho\left(E-E_{p}\right) e^{-i E\left(t-t^{\prime}\right)} f_{\alpha}(E) .
\end{aligned}
$$

In the above equations $E_{p}=2 t_{L} \cos (p \pi /(M+1))$ is the energy of the transverse channel $p$. These channels appear due to the width of the leads which in the tightbinding description is given by the number of the sites $M$ in the transverse direction. More exactly, the many channel lead is constructed by taking $M$ semiinfinite 1D leads and by coupling them through nearest neighbor hopping constants. Then $\chi_{p}\left(i_{\alpha}\right)=\sqrt{\frac{2}{M+1}} \sin \left(\frac{p i_{\alpha} \pi}{M+1}\right)$ is the transversal eigenfunction associated to $E_{p}$, and $\rho(E)$ is the density of states at the endpoint of a semiinfinite one-dimensional lead:

$$
\rho(E)=\theta\left(2 t_{L}-|E|\right) \frac{\sqrt{4 t_{L}^{2}-E^{2}}}{2 t_{L}^{2}} .
$$


Finally, $f_{\alpha}(E)$ is the Fermi function in the lead $\alpha$. The bias is included in our approach as the difference between the two chemical potentials of the leads $V=\mu_{L}-\mu_{R}$.
Plugging all these elements in the current formula one gets the main expression that will be numerically implemented in the next section:

$$
J_{\alpha}(t)=-\frac{2 e}{h} \operatorname{Im}\left(\sum_{p=1}^{M} \sum_{m_{\alpha}, n_{\alpha} \in C_{\alpha}} \int_{-2 t_{L}+E_{p}}^{2 t_{L}+E_{p}} d E \int_{0}^{t} d s e^{-i E(s-t)} \Gamma_{m_{\alpha}, n_{\alpha}}^{\alpha, p}(E ; t, s)\left(G_{m_{\alpha} n_{\alpha}}^{R}(t, s) f_{\alpha}(E)+G_{m_{\alpha} n_{\alpha}}^{<}(t, s)\right)\right) .
$$

We have introduced the energy and time-dependent quantity $\Gamma_{m, n}^{\alpha, p}$ that takes also into account the $M$ channels in the lead:

$$
\begin{aligned}
& \Gamma_{m_{\alpha}, n_{\alpha}}^{\alpha, p}(E ; t, s)=\sum_{i_{\alpha}, j_{\alpha}=1}^{M} \rho\left(E-E_{p}\right) \chi_{p}\left(i_{\alpha}\right) \chi_{p}\left(j_{\alpha}\right) \times \\
& V_{i_{\alpha} m_{\alpha}}(t) V_{j_{\alpha} n_{\alpha}}(s) .
\end{aligned}
$$

A similar formula can be written down for the current $J_{\beta}$ and therefore one defines as well the net current

$$
J(t)=J_{\alpha}(t)+J_{\beta}(t)
$$

It is important to observe that in contrast to the simple case of a single-site system the expressions for the two currents imply the Green functions at different contacts. The retarded and the lesser Green functions are then to be computed from the Dyson and Keldysh equations ${ }^{13}$ :

$$
\begin{aligned}
& G^{R}\left(t, t^{\prime}\right)=G_{0}^{R}\left(t, t^{\prime}\right)+\int_{0}^{t} d t_{1} G^{R}\left(t, t_{1}\right) \int_{0}^{t_{1}} d t_{2} \Sigma^{R}\left(t_{1}, t_{2}\right) G_{0}^{R}\left(t_{2}, t^{\prime}\right) \\
& G^{<}\left(t, t^{\prime}\right)=\int_{0}^{t} d t_{1} G^{R}\left(t, t_{1}\right) \int_{0}^{t^{\prime}} d t_{2} \Sigma^{<}\left(t_{1}, t_{2}\right) G^{A}\left(t_{2}, t^{\prime}\right),
\end{aligned}
$$

where $G_{0}^{R, A}\left(t, t^{\prime}\right)$ are the retarded and advanced Green functions of the isolated central region and $\Sigma^{R,<}$ are the retarded and lesser self-energies. $G_{0}^{R}\left(t, t^{\prime}\right)$ has a simple expression in terms of the discrete spectrum $\left\{E_{\lambda}\right\}$ of the central region and its localized eigenfunctions $\psi_{\lambda}$ (clearly $\lambda=1, . . N)$ :

$$
G_{0, m n}^{R}\left(t, t^{\prime}\right)=-i \theta\left(t-t^{\prime}\right) \sum_{\lambda} \psi_{\lambda}(m) \overline{\psi_{\lambda}(n)} e^{i E_{\lambda}\left(t-t^{\prime}\right)}
$$

We emphasize the lower integration limit $t=0$ in equations (16) and (17). This is due to the fact that there is no coupling for $t<0$. In the adiabatic setup the coupling is established in the remote past and one should set a lower cutoff in the numerical implementation. However, the Dyson equation still contains two coupled integrals. The two self-energies above contain the information from the leads and are finite rank matrices in the Hilbert space of the central region $S(\gamma=\alpha, \beta)$ :

$$
\begin{aligned}
& \Sigma_{m n}^{R}\left(t, t^{\prime}\right)=\sum_{\gamma} V_{\gamma}(t) g_{i_{\gamma}, j_{\gamma}}^{R}\left(t, t^{\prime}\right) V_{\gamma}\left(t^{\prime}\right) \delta_{m m_{\gamma}} \delta_{n n_{\gamma}} \\
& \Sigma_{m n}^{<}\left(t, t^{\prime}\right)=\sum_{\gamma} V_{\gamma}(t) g_{i_{\gamma}, j_{\gamma}}^{<}\left(t, t^{\prime}\right) V_{\gamma}\left(t^{\prime}\right) \delta_{m m_{\gamma}} \delta_{n n_{\gamma}}
\end{aligned}
$$

We stress that the indices of the leads' Green function are unambiguously determined as the neighbor sites of the contact surface $C_{\alpha}$. In the single channel case $M=1$ one recovers simpler expressions. In particular the retarded Green function of the lead can be expressed through the Bessel function of the first kind:

$$
g_{1_{\gamma}, 1_{\gamma}}^{R}\left(t, t^{\prime}\right)=\frac{-i \theta\left(t-t^{\prime}\right) J_{1}\left(2 t_{L}\left(t-t^{\prime}\right)\right)}{2 t_{L}\left(t-t^{\prime}\right)} .
$$

We point out the difference between the exact form of the retarded self-energy and the simple wide-band limit expression (which simplifies to $\delta\left(t-t^{\prime}\right)$ up to some constants). Note that the retarded Green function gives the leads' self-energy and is a highly oscillating functions. it will turn out in Section III that this behavior has crucial effects on the transient current. Another difficulty of Eq.(16) comes from the quadratic dependence of the selfenergies on the time-dependent coupling. Clearly this prevents any partial Fourier transform trick.

Given these, our strategy in solving the integral Dyson equation relies in transforming it into an algebraic equation of the form $A X=B$ where $A, X, B$ are generalized complex matrices depending on both spatial and time arguments. To this end we first plug the retarded self 
energy from Eq.(19) in the Dyson equation (16) and discretize the time arguments. Note that the variable $t_{2}$ is defined on a denser grid than the one used for $t_{1}$. The inner time integral is evaluated by a repeated 4-point Gauss method, which turned out to be accurate enough for the numerical results to be stable when increasing the number of integration steps. This procedure allows us to write the double integral as a matrix $\tilde{G^{R}} \tilde{A}$, where $\tilde{A}$ is actually a product of $G_{0}^{R}, \Sigma^{R}$ and some diagonal matrices containing the Gauss weights needed in the integration procedure. Then the adjoint of the generalized retarded Green function $\tilde{G}^{R}$ is simply the solution of the algebraic equation $(1-\tilde{A})^{*} \tilde{G}^{R^{*}}=\tilde{G}_{0}^{R^{*}}$. The true Green function is recovered by turning back the mixed indices of $\tilde{G}^{R}$. We stress that by solving the equation for $\tilde{G}^{R}{ }^{*}$ the Dyson equation is solved exactly. Moreover, no matrix inversion is required. This is certainly an advantage in the numerical simulations since it is known that matrix inversion is both memory and time-consuming. The advanced Green function is computed using the identity $G_{i j}^{A}\left(t, t^{\prime}\right)=G_{j i}^{R}\left(t^{\prime}, t\right)$ and the lesser Green function is derived from the Keldysh equation. Also, the timedependent occupation number can be computed as:

$$
N(t)=\operatorname{Im} \sum_{m \in S} G_{m m}^{<}(t, t) .
$$

The current in the right lead $J_{\beta}$ has a similar expression. We note that for a system with many sites one has to deal with different contact Green functions besides replacing only the Fermi function in the first term in the current formula. Moreover, in the transient regime the current conservation does not imply the usual identity $J_{\alpha}=-J_{\beta}$. We shall discuss this feature below.

In the Keldysh approach to time-dependent transport the problem is to extract physical information from the two contributions in Eq.(13). In the simplest case of a single-site and within the wide-band limit it was shown that the average current obeys a Landauer-like formula. The effects of a step-like or harmonic time-dependent potentials applied adiabatically on leads were studied both in the WBL 12 and beyond ${ }^{18}$. However, to our best knowledge no transient current calculation for a many-level structure beyond the wide-band limit has been performed within the Keldysh formalism.

\section{NUMERICAL SIMULATIONS}

In all the plots the bias, the energy, the hopping constants on the leads, the coupling strengths and the gate potentials will be expressed in terms of the hopping energy of the central region $t_{D}$ which is chosen as energy unit. The current is therefore given in units of $e t_{D} / \hbar$ and the time expressed in units of $1 / t_{D}$. Since the spectrum of the two-dimensional discrete Laplacian covers the range $\left[-4 t_{D}, 4 t_{D}\right]$ we shall take $t_{L}=2$ in order to match it to the spectral width of the one-dimensional lead $\left[-2 t_{L}, 2 t_{L}\right]$. We take also $e=\hbar=1$. The current given by Eq. (13) can be written as a sum of two contributions

$$
J_{\alpha}(t)=J_{\alpha}^{R}(t)+J_{\alpha}^{<}(t)
$$

the ' $<$ ' and ' $R$ ' labelling emphasizing that the corresponding term contains the lesser and retarded Green functions. We shall consider for simplicity only single channel leads.

\section{A. Single-site}
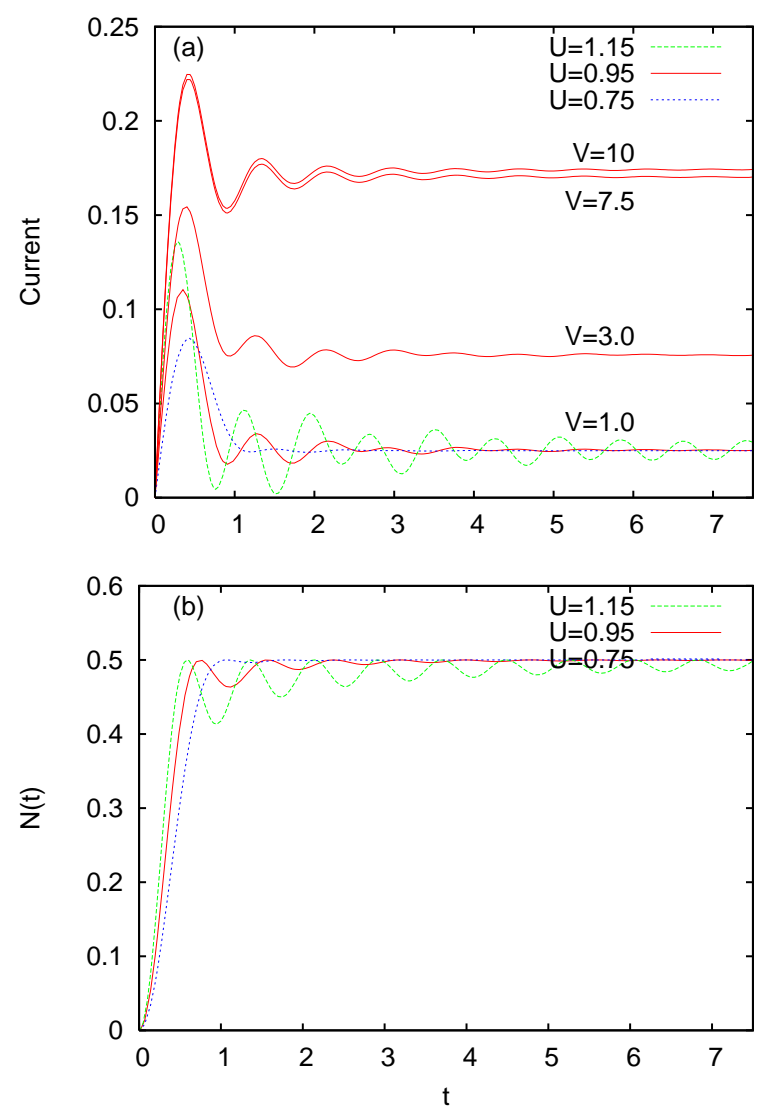

FIG. 2: (Color online) (a) The transient current for one site coupled to single-channel leads. We present curves for different values of the coupling strength $U$ and of the bias $V$. (b) The effect of the coupling amplitude $U$ on the occupation number. The bias is fixed to $V=1.0$, and $k T=0.0001$.

We start this section by discussing the case of a single site dot which allows qualitative discussion on the transient regime and on the transition towards the steadystate. The site is coupled to single-channel leads (i.e. $N=M=1$ ). Both leads are coupled suddenly to the system with the same strength $U$, i.e $V_{\alpha}=V_{\beta}:=U$. The bias is applied symmetrically on leads, i.e. $\mu_{\alpha, \beta}=$ $\mu_{0} \pm \mathrm{eV} / 2, \mu_{0}$ being the chemical potential of the unbiased leads. We observe that for $\mu_{0}=0.0$ the single eigenvalue 
of the isolated system $E_{0}=0.0$ is located in the middle of the bias window $W=\left[\mu_{0}-e V / 2, \mu_{0}+e V / 2\right]$. As we shall see later on, the position of the eigenvalues of the system within the bias window has important implications on the transient current. The free retarded Green function of the single site system is simply $G_{0}^{R}=-i$ but the full retarded Green function is still given by the $i n$ tegral Dyson equation and an analytic solution is not at hand.

Fig. 2(a) gives the transient current for different values of the bias $V$ and of the coupling amplitude $U$ and reveals that the parameter that controls the shape and the amplitude of the oscillation is the coupling strength $U$. At moderate coupling $U=0.75$ the steady state (SS) is achieved fast but an oscillatory behavior is observed at $U=0.95$. The case $U=1.15$ is beyond the perturbative regime and the steady-state is not achieved in the selected time-range. As the bias increases the current saturates for values of $V$ that exceed the spectrum of the leads (i.e. for $V>8$ ), emphasizing the non linear transport regime. In turn, the bias neither affects the amplitude nor the period of the oscillations. This is due to the fact that in our model, as in all approaches based on the Keldysh formalism, there is no term in the Hamiltonian to describe the voltage drop across the sample, the bias being included only via the Fermi functions of the leads.

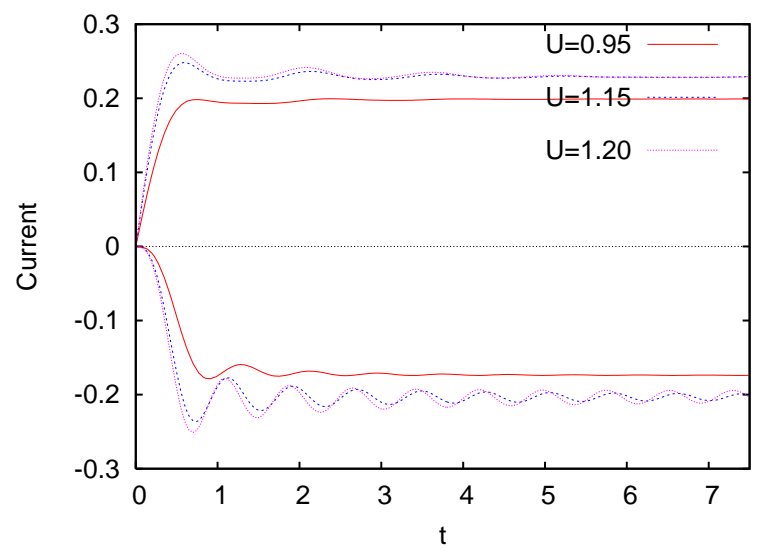

FIG. 3: (Color online) The two contributions to the transient current in the left lead. $J_{\alpha}^{R}(t)$ is always positive while the lesser contribution $J_{\alpha}^{<}(t)$ is negative. The chosen values for the coupling strength $U$ are given in the figure. The bias $V=1.0$, and $k T=0.0001$.

In Fig. 2(b) we plot the occupation number of the resonant site $N(t)$ for the same parameters as in Fig. 1(a). The behavior w.r.t. $U$ is similar. Few more things worth to be noticed: i) In the steady state regime the occupation of the site is $1 / 2$; ii) as $U$ increases while the bias stays constant the occupation number reaches its maximum value faster and the value corresponding to the steady state decreases; iii) Comparing Fig. 2(a) and 2(b) it can be seen that there is no clear relation between the principal maximum of the current and the one of the oc- cupation number; in fact the electrons are accumulating in the system even after the current starts to decrease towards the steady state.

Fig. 3 shows the two contributions to the current $J_{\alpha}^{R}$ and $J_{\alpha}^{<}$for $V=1.0$ and several coupling constants considered in Fig. 2. A physical significance of these two currents was proposed in Ref. 12 for the single site case. Although both Green functions at the contacts appearing in the current formula are 'dressed' by the leads' selfenergy one could view $J_{\alpha}^{R}$ as the current flowing towards the sample and $J_{\alpha}^{<}(t)$ as the current from to sample to the lead $\alpha$. One notices that the currents have opposite signs. Another observation is that the lesser contribution is responsible for the total current oscillations since $J_{\alpha}^{R}$ saturates quickly. However at small times $J_{\alpha}^{R}$ grows faster than $J_{\alpha}^{<}$, leading thus to the fast increase of the transient.

In order to understand the nature of the oscillations in the transient current and their dependence on the coupling strength $U$ it is useful to rewrite the current formula Eq. (13) in a more useful form (since we consider a single site system there is only one contact site and the indices of the Green functions can be omitted):

$$
J_{\alpha}(t)=-2 U^{2} \operatorname{Im} \int_{0}^{t} d s\left(G^{R}(t, s) F_{1}(s, t)+G^{<}(t, s) F_{2}(s, t)\right),
$$

where $F_{1}, F_{2}$ are two oscillating integrals:

$$
\begin{aligned}
& F_{1}(s, t)=\int_{-2 t_{L}}^{2 t_{L}} d E f_{\alpha}(E) \rho(E) e^{-i E(s-t)} \\
& F_{2}(s, t)=\int_{-2 t_{L}}^{2 t_{L}} d E \rho(E) e^{-i E(s-t)}
\end{aligned}
$$

One can easily observe that actually $F_{2}(s, t)$ can be expressed through Bessel function of the first kind:

$$
F_{2}(s, t)=\frac{\theta(t-s) J_{1}\left(2 t_{L}(t-s)\right)}{2 t_{L}(t-s)} .
$$

For fixed $t, F_{2}$ is an oscillating function of $s$ whose oscillation amplitudes increase with $s . F_{1}$ does not have a simple analitical expression but it has a similar behavior.

The oscillatory behavior of the current is clearly decided by the convolution in Eq. (24). Besides the oscillations of $F_{1}$ ans $F_{2}$ one expects as well a complex behavior of the Green functions. We recall that the Dyson equation counts the infinite back-and-forth tunneling processes involving the leads and that the amplitudes of these events are even powers of $U$. Now, the higher order terms in the Dyson equation contain multiple integrals of products of the leads' self-energy which is highly oscillating (see (21)) Therefore if $U<<1$ there will be only few low-order significant contributions from the complicated lead-sample scattering. The critical value $U=1.00$ corresponds to the onset of the nonperturbative regime, and the method we use for solving the Dyson equation captures as well this situation, taken into account all contributions. 

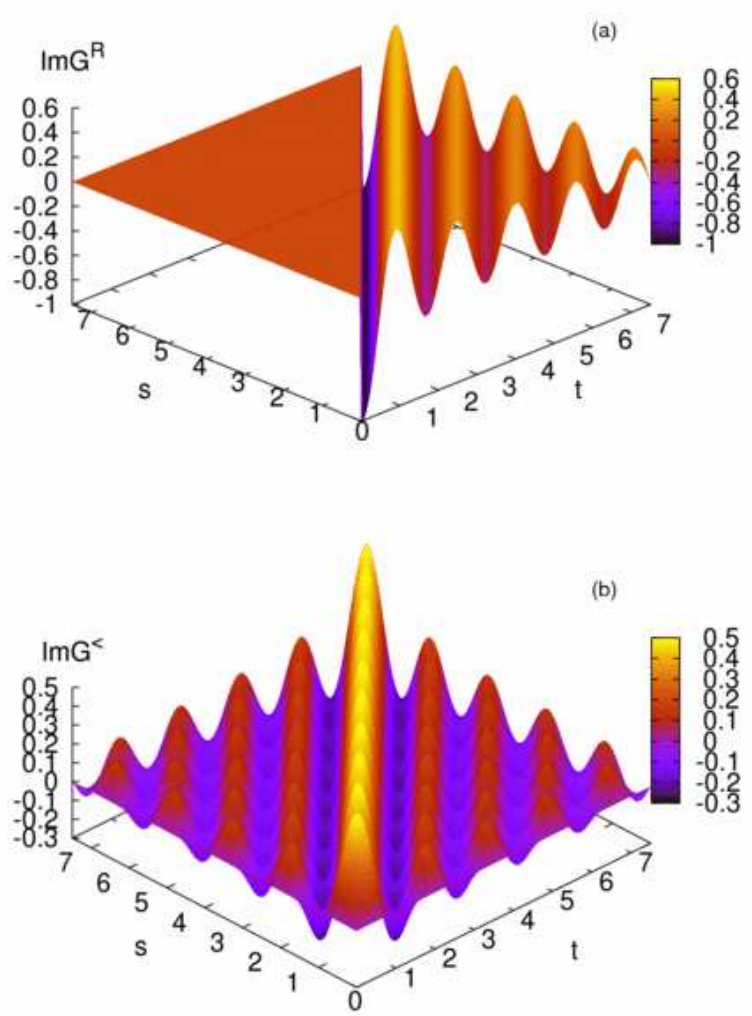

FIG. 4: (Color online) The imaginary parts of the retarded (a) and lesser (b) two-time Green functions of the coupled single site. The bias $V=1.0$ and the coupling strength $U=1.2$. The oscillations seen along the 'diagonal' in (b) corresponding to almost equal times are responsible for the oscillations of the occupation number.

We give in Fig. 4 the 3D plots of the imaginary parts for the retarded and lesser Green functions at coupling strength $U=1.20$, which leads to oscillations of the transient. These are the relevant quantities in the current formula since it turns out that the real part of $G^{R}$ and of $F_{2}$ are vanishingly small (not shown).

One observes that $G^{R}(t, s)=0$ for $s \geq t$ and, more interestingly, that $G^{R}(t, s)$ and $G^{<}(t, s)$ exhibit pronounced oscillations as time varies and reach a limit value as $s$ approaches $t$. In the case of the retarded Green function this limit is constant and equals $-i$, which is simply the value of the unperturbed retarded Green function. This feature is easy to understand by looking at the Dyson equation and noticing that when $s \rightarrow t$ the integration range of the inner integral shrinks considerably so that at almost equal times the perturbed Green function resembles the unperturbed one. This argument is not restricted to the single site case we are discussing. Also, since the spectrum of the discrete Laplacian is symmetric, the unperturbed retarded Green function will always be real and therefore the real part of the full Green function will always be vanishingly small, as we shall check
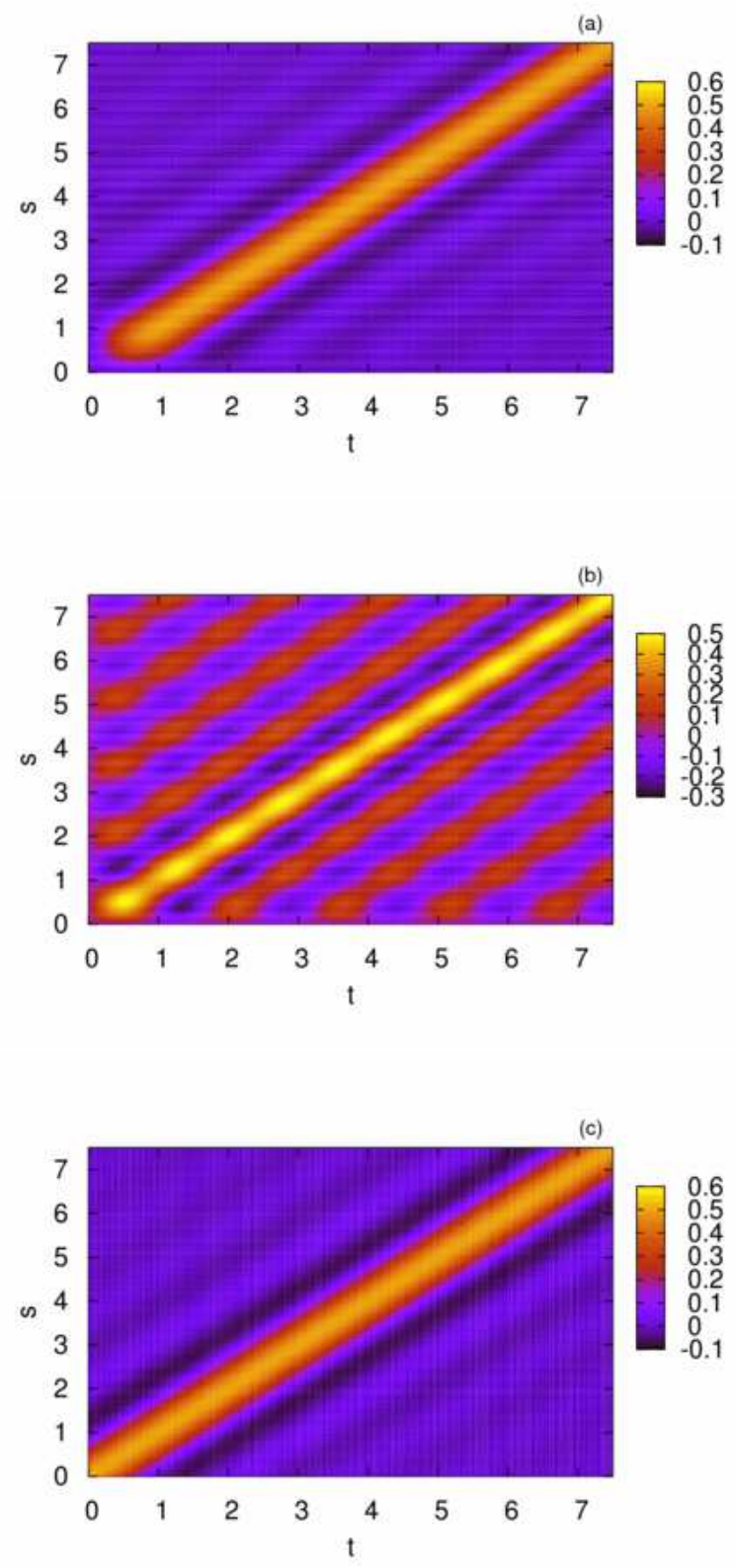

FIG. 5: (Color online) The imaginary part of the lesser twotime Green function for the moderate coupling $U=0.75$ (a) and strong coupling $U=1.2$ (b). (c) The real part of the oscillating function $F_{2}$. The bias $V=1.0$.

numerically in the many site case. In contrast, the limit of $\operatorname{Im} G^{<}$as $s \rightarrow t$ is not a constant w.r.t. $t$ but shows oscillations that disappear as $t$ increases. In the particular single-site case the limit value of $G^{<}$is clearly the occupation number of the site, whose oscillations were shown already in Fig. 2(b).

Figs. 5 (a) and (b) show 3D maps of the imaginary part of the lesser Green function and emphasize the role of the coupling strength on the transient. At moderate coupling 

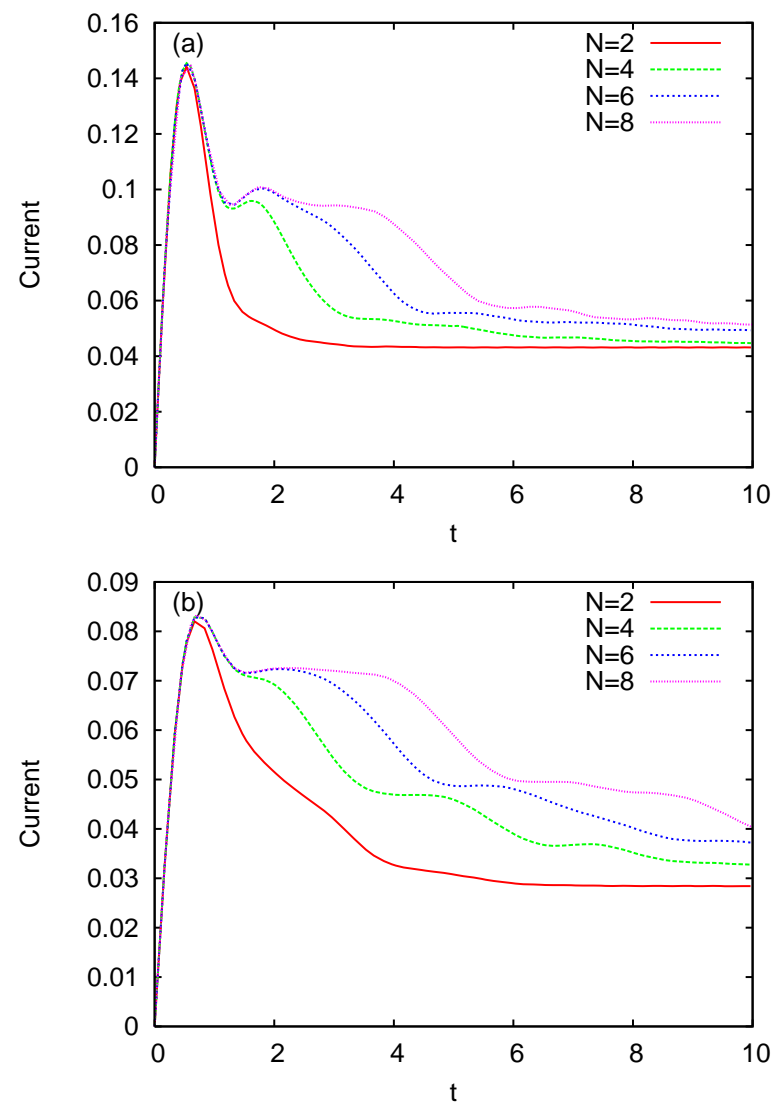

FIG. 6: (Color online) (a) The transient current in the left lead $J_{\alpha}(t)$ for different sizes of the $1 \mathrm{D}$ central region. The number of sites $N$ is indicated in the figure. In (a) the coupling to the leads is $U=0.75$ and in (b) $U=0.50$. By decreasing $U$ the shoulders in (a) turn to clear steps in (b). The bias is fixed to $V=2.0$, and $k T=0.0001$.

$U=0.75$ one observe small amplitude oscillations except for $s \sim t$, in clear contrast to the case $U=1.20$ where pronounced oscillations exist even for large time differences. Inspecting the real part of the function $F_{2}$ given in Fig. 5 (c) we see that it does not depend on $t$ when $s \sim t$ and that this gives the main contribution to the integral (24). It is now clear from Figs. 5 (a) and (c) that the corresponding current will be nearly stationary once the sample is charged (i.e. for $t>0.75$ ), because by increasing $t$ the 'off-diagonal' contributions are very small (some cancelations being possible as well). When $U$ increases the integral will collect instead nonnegligible contributions from the entire range $(0, t)$ and therefore the current will oscillate. These observations lead to the following statement: The steady state will be achieved at instant $t_{s}$ if for any $t>t_{s}$ there are no contributions for long-time differences, i.e. when both contact Green functions $G^{R}(t, s)$ and $G^{<}(t, s)$ vanish for $s>t_{s}$. It is easy to observe that this condition implies the well known criteria for the steady state $G(t, s)=G(t-s, 0)$.

\section{B. Many-site case.}
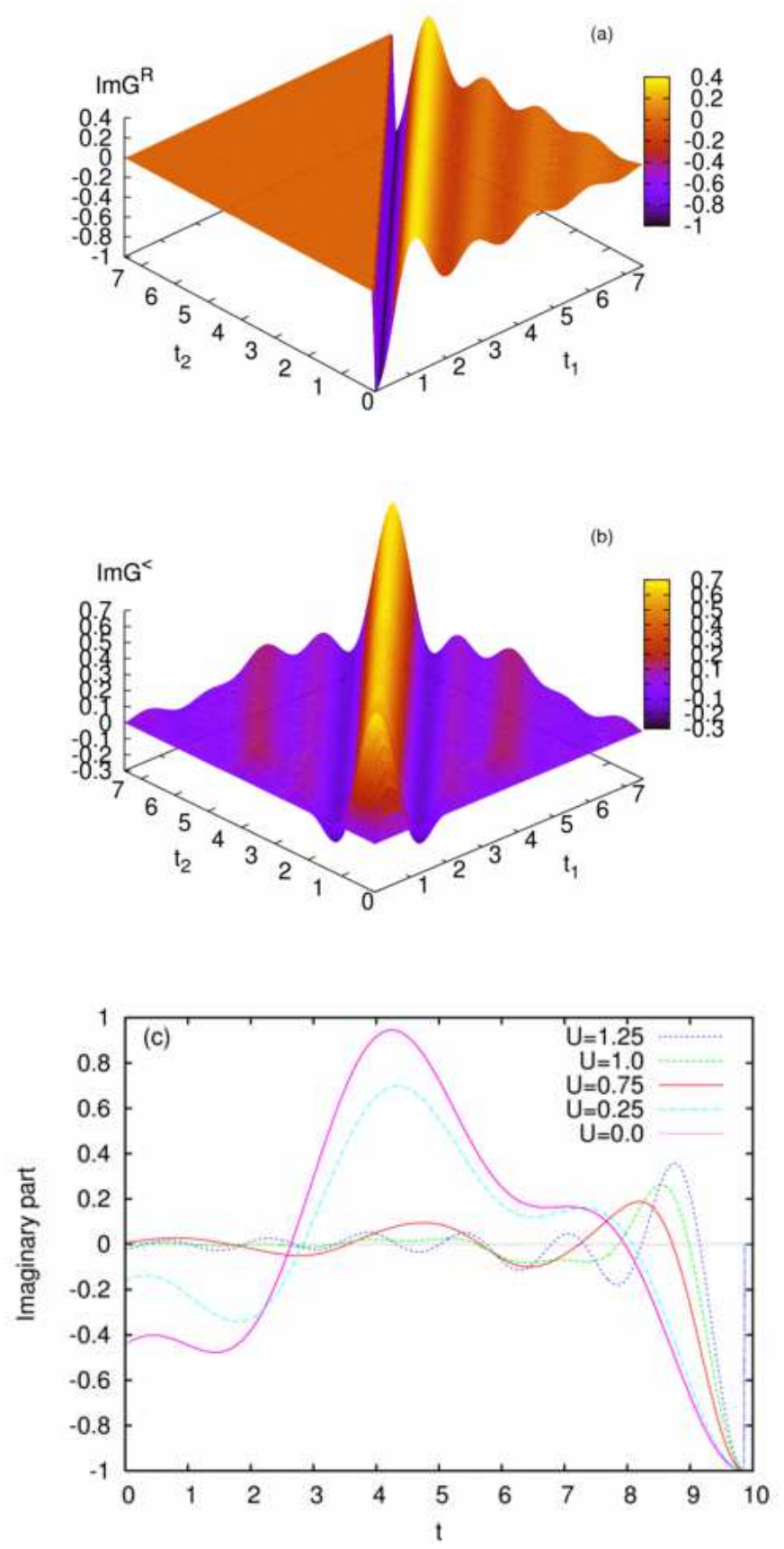

FIG. 7: (Color online) The imaginary part of the left contact retarded Green function (a) and of the lesser Green function for the 4 site system (b). (c) The imaginary part of $G_{11}^{R}\left(t_{1}=10, t_{2}\right)$ at different couplings $U$. At small coupling $\operatorname{Im} G^{R}$ resembles the retarded Green function of the isolated system (the curve corresponding to $U=0.0$ ).

We consider now the more interesting case where the central region has more than one level. Fig. 6(a) emphasizes the qualitative differences between the transients 
of $1 \mathrm{D}$ systems with $N=2,4,6,8$ sites. A general feature is that as the size increases the transient develops a 'shoulder' which is not met in the single site case. For $N=2$ a second smaller slope of decrease is noticed for $t \in[1.6: 2.5]$. For $N=4$ the system experiences few very different regimes before reaching the steady state. It first decreases faster up to $t \sim 1.25$. For a short time a small hill develops around $t \sim 2$ then the decrease continues but clearly at a smaller rate. Finally, for $t>3.25$ the current approaches the steady state very slowly. A similar behavior is observed for $N=6$ and $N=8$, the main difference being that the 'shoulder' is longer and the intermediate slope is smaller. The patterns described above suggest that there are some intermediate regimes, some of them being characterized by a rather stable current. Fig. 6(b) emphasizes that at lower coupling $U=0.50$ the transient is even smoother and for $N=4,6$ and 8 one notices the formation of clear steps.

When the coupling strength $U$ is increased the transient shows oscillations but they are fewer than in the single site (not shown). Since the two oscillatory integrals over energy in the current formula and both selfenergies do not depend on the number of sites in the system the above size effects should be explained only by the behavior of the contact Green functions. We show in Fig. 7(a) and (b) the imaginary parts of the the contact Green functions $G_{11}^{R,<}$ of the 4 site system for a strong coupling $U=0.25$ (it turns out again that the real part of $G_{11}^{R}$ is vanishingly small). Comparing with Fig. 4 it is obvious that for the 4 site dot the Green functions have a more regular behavior and in particular the occupation number of the contact site 1 shows milder oscillations than the occupation number of the single site. Fig. 7(c) gives the $\operatorname{Im} G_{11}^{R}\left(t_{1}=10, t_{2}\right)$ as a function of $t_{2}$ for different couplings and reveals that at weak coupling to the leads the full retarded Green function is close to the unperturbed one and the electron dynamics inside the system must resemble the one of the isolated sample. Indeed, the curve at $U=0.25$ follows the oscillations of the free Green function which is also given in the figure (the curve corresponding to $U=0.0$ ). We plot the imaginary part since it turns out again that the real part is vanishingly small so it does not contribute considerably to the retarded current. As $U$ increases the Green function changes and shows clear oscillations imposed by the leads' self-energy.

The analysis performed so far was focused on the behavior of the transient current as the intrinsic parameters of the system (i.e. its size and the height of the tunneling barriers at the contacts are varied. Nevertheless, in a typical transport experiment these parameters are fixed and one usually measures the current by varying the bias or a plunger gate voltage. From steady-state current measurements it is well known that the role of such a gate potential is to bring one or more levels of the quantum dot within the bias window (BW). We show in what follows that at fixed bias and given coupling strength to the leads one can tune the transient current with a gate po- tential. Moreover, by inspecting the transient behavior as the gate potential is varied, it is possible to extract some information about the number of states within the bias window or above it. We will make the discussion for the 4 site dot. The gate potential is simulated by the diagonal term $V_{g}$ added to the on-site energy of the system. We fix the bias window to $\mathrm{W}=2.0$ and for convenience we set $\mu_{R}=0.0$. Fig. 8 (a) give a families of transients for coupling strength $U=0.75$ and various values of $V_{g}$ specified in the figures. Fig. 8(b) shows the four levels of the isolated quantum dot as the gate potential scans the range [-4:4]. The $V_{g}$ values chosen in Fig. 8(a) corresponds to different location of the levels w.r.t. to BW. The bottom curve is irregular and settles down to a vanishing current because in this case there is no level within the BW. We note however that a nonvanishing transient current still develops shortly after the coupling is established. At $V_{g}=-1.0$ the highest level is located in the BW and the transient is smooth and already shows the additional shoulder noticed previously. The same thing happens when two states lie in the BW (at $V_{g}=0.0$ ), the difference being that the steady state current increases considerably. For $V_{g}>0.50$ it is clear from the structure of the spectrum that one cannot have more than two states in the BW and that the levels pass gradually above it. We found interesting to look at the transient currents for those gate potentials that still allow two states in the transmission range while pushing one or two states above BW. One notices that for $V_{g}=1.0$ the steady state currents do not distinguish the different spectral structure involved in transport, while the transient current is very sensitive to it.

In the case of the six site QD the shoulder in Fig. 4 is more pronounced because at $V_{g}=0.0$ there are exactly three states inside the bias window. We want to point out that since we have neglected the Coulomb interaction our simulations cannot capture the transport through excited states of the quantum dot. Tunneling processes involving such states would lead to a minipeak structure of the current maxima. We can consider however our results should describe qualitatively the transport involving more levels because for small dots the bias required to cover the ground state of $N$ electrons is much higher that the excitation energies.

Now we investigate two more features of the transient regime: The time-dependent charge filling of the central region and possible effects due to different shapes of the system or of the various ways in which one can couple the leads. Besides the 4 site 1D system discussed so far we consider also a $2 \times 2$ quantum dot. Both systems are submitted to the same bias and have equal coupling to the leads. However, in the case of the 2D quantum dot one can use different contacts for plugging the leads. We discuss two situations: i) A symmetric configuration in which the leads are attached to the opposite corners of the system, namely at the sites 1 and 4 and ii) An asymmetric coupling when we use the 1st and 3th sites as contacts. Fig. 9(a) reveals the changes induced in the 

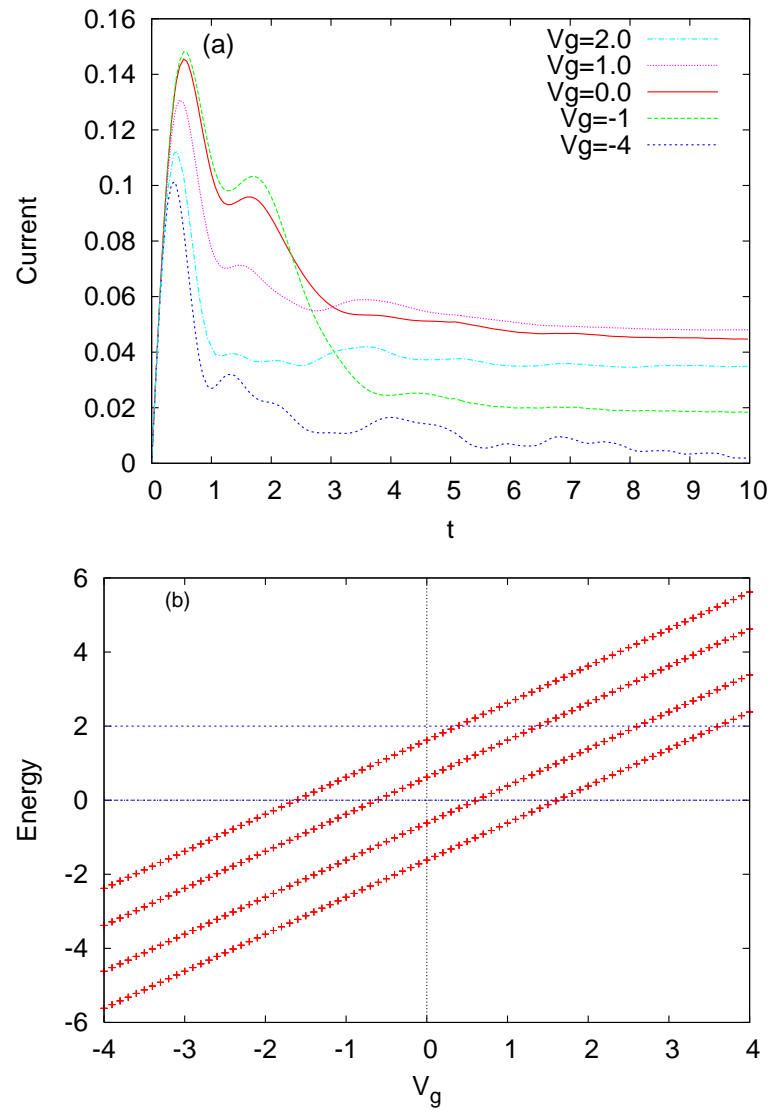

FIG. 8: (Color online) (a) The transient current for different values of the gate potential applied on the 4 site quantum dot for $U=0.75$. (b) The spectrum of the system as function of the gate potential. It can be checked that the additional shoulders observed in (a) develop when there is at least one state of the QD in the bias window and no state above it. Other parameters: $V=2$, and $k T=0.0001$. Note that the bias is applied asymmetrically, that is $\mu_{\beta}=0.0$.

transient curves in each case. The remaining subfigures show the occupation number $N_{i}(t)$ of each site $i=1,4$ for the $1 \mathrm{D}$ system and the two configurations considered. Inspecting Fig. 9(a) and (b) we see: i) The contact sites 1 and 4 are the first to be populated due to their proximity to leads; ii) Since $\mu_{\alpha}>\mu_{\beta}$ the right contact site (the 4th) gains less charge at a lower rate than the left contact (the first). Both $N_{1}$ and $N_{4}$ show a step-like behavior for a short period (around $t=1$ ). This coincides with the increased occupation number on the middle sites. We note also that the step in the occupation of the contact site $N_{1}$ ends when it is equaled by $N_{2}$. All the sites are then continuously filled up to the steady-state value. The occupation number on the right contact is smaller than the other ones which attain roughly the same value 0.65 . iii) The step-like behavior of the transient currents in the range $[1: 1.75]$ corresponds to the almost constant population of the contact sites in the same interval. The symmetric configurations is still characterized by a smooth transient but we notice that the step appears now later
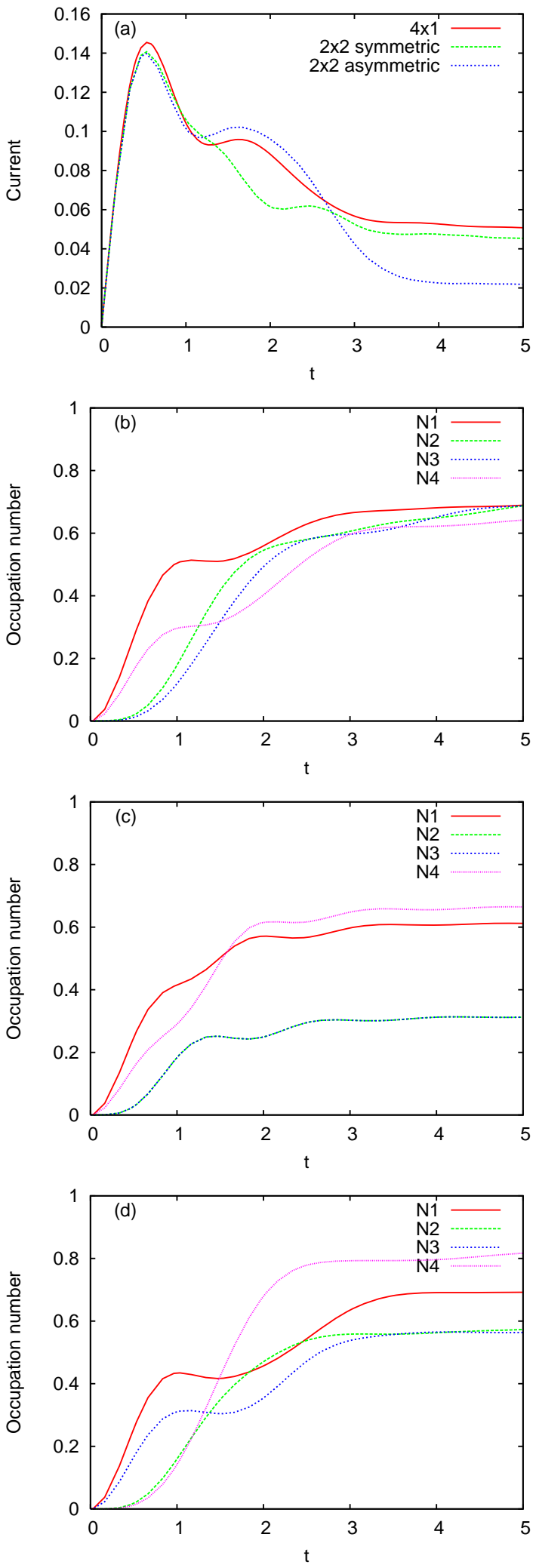

FIG. 9: (Color online) (a) The transient in the left lead $J_{\alpha}(t)$ for the $4 \times 1$ system and for the $2 \times 2$ system in the two configurations of the leads as mentioned in the text. (b) 1D system, (c) - symmetric configuration, (d) - asymmetric The occupation numbers $N_{i}(t)$ of the $i$-th site for the three cases considered in Fig. 8(a). Other parameters: $U=0.75$, $V=2.0$, and $k T=0.0001$. 


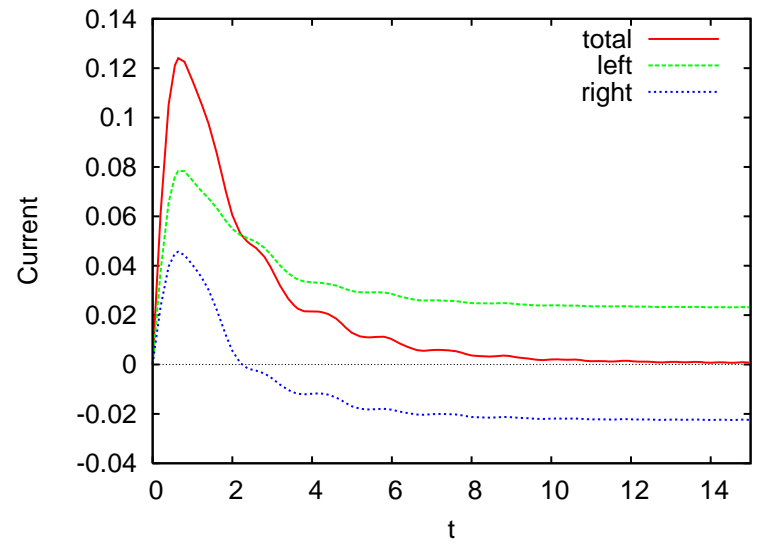

FIG. 10: (Color online) The total transient current and the components in the left lead and right lead. Other parameters: $U=0.5, V=2, k T=0.0001$.

that in the 1D case. Fig. 9(c) confirms again that this stable regime is assigned to a constant flow in the contact site. Also it reflects that the charge is equally distributed in the sites 2 and 3 which are located symmetrically w. r.t. the leads. In the asymmetric geometry the transient is rather similar to the one of the $1 \mathrm{D}$ system up to $t=2.8$ but then drops to a lower steady state value. The occupation numbers show that the fourth site carries more charge than the contact sites in the steady state. This means in our opinion that part of this charge simply accumulates and is not participating in transport. More interestingly, we note that in contrast to the symmetric geometry $N_{2}$ and $N_{3}$ are different in the transient regime but reach the same value in the steady state. We mention that time-dependent simulations were performed recently in the case of an Aharonov-Bohm ring starting from the Schrödinger equationi. 21

We discuss now briefly the total current $J_{\alpha}+J_{\beta}$ which is given in Fig. 10 along with its two components (the + sign is due to the fact that the current $J_{\beta}$ represents the current from the lead $\beta$ to the system and therefore has opposite sign). As already mentioned, only in the steady state the current conservation reads as $J_{\alpha}=-J_{\beta}$. Consequently, the net current $J_{\alpha}+J_{\beta}$ vanishes. However, in the transient regime the two currents, although having similar shape differ significantly.

To substantiate further the previous analysis we present in Fig. 11(a) the contour plot of the current as function of time and gate potential for the $2 \times 2$ site quantum dot coupled to the leads in the symmetrical configuration. Fig. 11(c) gives the spectrum of the system as the gate potential varies. The middle eigenvalue is doubly degenerated. When the levels are either below or above the bias window ( $W=1.0$ starting from $\mu_{R}=0.0$ ) the transient oscillates for quite a long time before passing to the steady state. We also observe that in these two extreme limits the transient oscillations are qualitatively different. For $V_{g} \sim-4$ the current shows
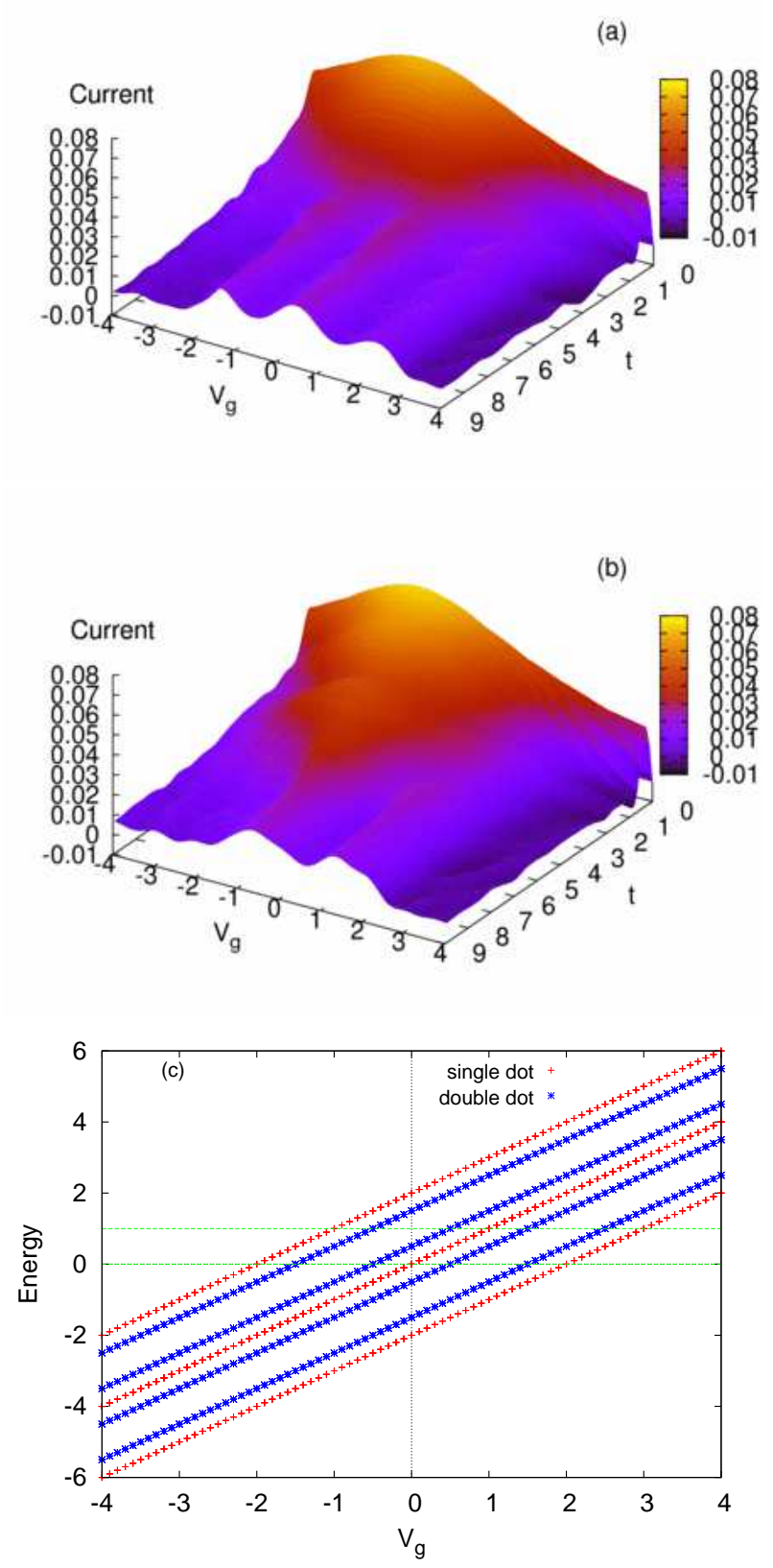

FIG. 11: (Color online) (a) The 3D map of the transient current for a $2 \times 2$ site QD as a function of time and gate potential $V_{g}$. The maxima are related to the spectrum of the isolated system given in (c). (b) The 3D map of the transient current for $2 \times 2$ the double dot $t_{24}=t_{13}=0.5$ Other parameters: $V=1.0, U=0.50, k T=0.0001$. (c) The spectra of the two systems as a function of the gate potential. The lines mark the bias window.

decreasing oscillations towards the steady state. In contrast, for $V_{g} \sim 4$ one remarks faster oscillations and more importantly, negative values of the transient. Since in this regime all the levels are above the bias window and there is no way to pass electrons to the right lead it is 
clear that the negative current in the left lead is just the reflected one. We underline that this effect is due to the fact that we have considered a finite spectral width of the leads and that similar features were reported for the single site case $^{18}$. As expected, as the system approaches the stationary regime the current shows three maxima associated to the passage of the localized levels through the bias window. Actually the levels turn to resonances when coupling the leads to the system but since one has to deal with a time-dependent Hamiltonian it is difficult to characterize the location and width of these resonances in the transient regime. This is why in the 3D plot one cannot distinguish between different resonances at times $t<3.0$. Fig. 11(b) presents the transient current for the same $2 \times 2$ system except that the hopping parameters $t_{13}$ and $t_{24}$ are reduced to 0.5 . In this case one can view the system as a double dot, each dot composed of two sites. As the spectrum from Fig. 11(b) shows, the degeneracy is lifted and the level spacing diminishes. As a consequence in the long time regime one gets two broader peaks, since the four levels are now grouped into pairs.

All the features presented above emphasize that the transient regime of the many-level structures is quite different from the single-level system.

\section{CONCLUSIONS}

We have performed transient current calculations for a many-level finite system coupled suddenly to semiinfinite biased leads. Our method is based on the nonequilibrium Green-Keldysh machinery. We find numerically an exact solution of the integral Dyson equation which is solved as an algebraic equation. By analyzing the behavior of the retarded and lesser Green functions we explain qualitatively the shape of the transient current and the passage to the steady state. The amplitude of the coupling to the leads controls essentially the convergence to a steady state. We have identified non-trivial effects of the many-level structure of the system and presented an intuitive picture of the charge filling by studying the occupation number inside the system. By increasing the system size the shape of the transient current and the evolution towards the steady state differs significantly from the single-site oscillatory behavior and depends crucially on the number of electronic states available in the bias window. We predict that a step-like structure could be observed in transient current measurements by applying a gate potential on the system that tunes the higher levels within the bias window. Different transients are expected to appear as well when different coupling geometries of the leads are used.

The present method can be used for studying the response of mesoscopic systems to more complicated timedependent couplings to the leads: pulses having different lengths and decaying rates, and non-periodic signals.

\section{Acknowledgments}

This work was supported in part by the research programme of the Icelandic Reaserch Council for Nanoscience. V.M was also supported by CEEX Grant D11-45/2005. We acknowledge useful discussions with C. S. Tang.
1 M. Switkes, C. M. Marcus, K. Campman, and A. C. Gossard, Science 283, 1905 (1999).

2 L. J. Geerligs, V. F. Anderegg, P. A. M. Holweg, J. E. Mooij, H. Pothier, D. Esteve, C. Urbina and M. H. Devoret, Phys. Rev. Lett. 64, 2691 (1990).

3 P. W. Brouwer, Phys. Rev. B 58, R10135 (1998).

4 O. Entin-Wohlman, A. Aharony, and Y. Levinson, Phys. Rev. B 65, 195411 (2002).

5 M. Moskalets and M. Büttiker, Phys. Rev. B 66, 205320 (2002).

${ }^{6}$ L. Arrachea, Phys. Rev. B 72, 125349 (2005).

7 L. Arrachea, M. Moskalets, Phys. Rev. B 74, 245322 (2006).

8 G. Platero and R. Aguado, Physics Reports 395, 1 (2004).

9 J.E. Avron, A. Elgart, G.M. Graf, and L. Sadun, Comm. Pure and Appl. Mathematics LVII, 0538 (2004).

10 W.G. van der Wiel, T.H. Oosterkamp, S. de Franceschi, C.J.P.M. Harmans, and L.P. Kouwenhoven. Strongly Correlated Fermions and Bosons in Low-Dimensional Disordered Systems, eds. I.V. Lerner et al., pp.43-68 (2002), Kluwer Academic Publishers.

11 C. Caroli, R. Combescot, P. Nozieres, and D. Saint-James, J. Phys. C 4, 916 (1971).

12 A.-P. Jauho, N. S. Wingreen, and Y. Meir, Phys. Rev. B
50, 5528 (1994).

13 H. Haug, A.-P. Jauho, Quantum Kinetics in Transport and Optics of Semiconductors (Springer, Berlin, 1996).

14 T Fujisawa, D G Austing, Y Tokura, Y Hirayama and S Tarucha, J. Phys. Cond. Matt. 15 R1395 (2003).

15 L. P. Kouwenhoven, A. T. Johnson, N. C. van der Vaart, C. J. P. M. Harmans, and C. T. Foxon, Phys. Rev. Lett. 67, 1626 (1991).

16 W. Aschbacher, V. Jaksic, Yan Pautrat C-A. Pillet, Lecture Notes in Mathematics 1882/2006 Springer Berlin.

17 G. Nenciu, http://xxx.lanl.gov math-ph/0610074 to appear in J. Math. Phys.

18 Y. Zhu, J. Maciejko, T. Ji, H. Guo, and J. Wang, Phys. Rev. B 71, 075317 (2005), J. Maciejko, J. Wang, and H. Guo, Phys. Rev. B 74, 085324 (2006).

19 S. Kurth, G. Stefanucci, C.-O. Almbladh, A. Rubio, and E. K. U. Gross, Phys. Rev. B 72, 035308 (2005), G. Stefanucci, C.-O. Almbladh Phys. Rev. B 69, 195318 (2004).

${ }^{20}$ G. Stefanucci, S. Kurth, A. Rubio, E. K. U. Gross, cond-mat/0701279.

21 B. Szafran and F. M. Peeters Phys. Rev. B 72, 165301 (2005).

22 J. Rammer, H. Smith, Rev. Mod.Phys 58, 323 (1986). 